\title{
Mobile eHealth Platform for Home Monitoring of Bipolar Disorder
}

\author{
Joan Codina-Filbà ${ }^{10000-0003-1311-1693]}$, Sergio Escalera ${ }^{2,4[0000-0003-0617-8873],}$ \\ Joan Escudero ${ }^{3}$, Coen Antens ${ }^{2}$, Pau Buch-Cardona ${ }^{2,4[0000-0002-0031-1285]}$, and \\ Mireia Farrús ${ }^{1,4[0000-0002-7160-9513]}$ \\ 1 Universitat Pompeu Fabra, Barcelona, Spain \\ \{joan.codina,mireia.farrus\}@upf .edu \\ 2 Computer Vision Center, UAB, Cerdanyola del Vallès, Spain \\ \{sergio.escalera, coen, pbuch\}@cvc.uab.cat \\ 3 Pulso Ediciones SL, Sant Cugat del Vallès, Spain \\ j.escudero@pulso.com \\ 4 Universitat de Barcelona
}

\begin{abstract}
People suffering Bipolar Disorder (BD) experiment changes in mood status having depressive or manic episodes with normal periods in the middle. $\mathrm{BD}$ is a chronic disease with a high level of non-adherence to medication that needs a continuous monitoring of patients to detect when they relapse in an episode, so that physicians can take care of them. Here we present MoodRecord, an easy-to-use, non-intrusive, multilingual, robust and scalable platform suitable for home monitoring patients with $\mathrm{BD}$, that allows physicians and relatives to track the patient state and get alarms when abnormalities occur.

MoodRecord takes advantage of the capabilities of smartphones as a communication and recording device to do a continuous monitoring of patients. It automatically records user activity, and asks the user to answer some questions or to record himself in video, according to a predefined plan designed by physicians. The video is analysed, recognising the mood status from images and bipolar assessment scores are extracted from speech parameters. The data obtained from the different sources are merged periodically to observe if a relapse may start and if so, raise the corresponding alarm. The application got a positive evaluation in a pilot with users from three different countries. During the pilot, the predictions of the voice and image modules showed a coherent correlation with the diagnosis performed by clinicians.
\end{abstract}

Keywords: bipolar disorder $\cdot$ eHealth $\cdot$ Mobile monitoring $\cdot$ Data fusion.

\section{Introduction}

Bipolarity - or bipolar disorder - is one of the most common forms of mental illness, which is characterised by episodes combining euphoria and depression phases, becoming usually a persistent disorder with a prevalence in the population of about $1 \%$ [25]. During bipolarity episodes, different aspects of the 
patient life become altered. Sleeping hours, for instance, increase in depression or decrease in manic states while sociability and sexual interest behave inversely. The detection of incipient changes on mood (prodromes) when patients are euthymic (normal status), allow clinicians to do psychological treatments designed for relapse prevention in conjunction with mood stabilisers [4]. Prodromes are defined as "any cognitive, behavioural and affective signs or symptoms that may make patients think they are entering an early stage of an episode" [16]. While objectively a patient may be able to detect prodromes [17], a high rating is not able to seek treatment at an early stage of future relapse [15].

The treatment of bipolarity faces several difficulties. On the one hand, the high level of non-adherence to medication; some patients, because they like the positive feelings of the 'highs' they have in manic episodes; others, because the idea of an indefinite treatment discourages them [17]. Another problem is that, once started, both mania and depression episodes produce a positive feedback that fuels themselves: at the early stages of mania, the increased sociability and less need to sleep arouses more social contacts and sleep disorders. The first stages of depression create a loss of interest in people, disrupting social contact, reducing activity and increasing laziness and sleeping time. These difficulties are reasons to make a close follow-up of the subject in order to detect the prodromes and tackle them. In addition, the chronic condition and the time changing mood with intermittent episodes of mania or depression, with normal episodes in between, makes the bipolar disorder a good candidate to develop a home monitoring tool to observe changes in patients behaviour and mood status to raise the corresponding alarms when mood destabilises in any sense.

The current health care environment demands an objective assessment of the patient state. While in many branches of medicine this can be performed using the corresponding biomarkers, this is not the case of psychiatry where the evaluation is based on an interview with the patient. An objective measure of the patient state is crucial to perform an accurate monitoring of the patient evolution and to interchange information between clinicians. Rating scales (RS) allow to obtain these objective measurements in a quantitative way. Many RSs have been validated to be reliable - different evaluators will produce consistent results for a given subject - and are able to produce a valid diagnosis. A RS is composed of different items to evaluate with a corresponding rubric indicating how to measure it. The combination of the scores of the items gives the final score, which becomes the objective measurement of the the patient's state. There are self-administered scales thought to be filled by the user herself and clinicianadministered scales filled by physicians.

The evaluation of the patients suffering BD is based on two different RSs: one to account for the severity of depression and the other for mania. The most commonly used scales are the Hamilton Depression Rating Scale (HDRS) [14], and the Young Mania Rating Scale (YMRS) [31]. Both scales require clinicians to evaluate and rate different aspects of the patient related to prodromes. Although the scales measure opposite states, they assess alterations in similar items (but in different sense), e.g. changes in physical activity, sleep, speech or sexual interest. 
Automated and objective measures of these prodromes would simplify and shorten clinicians task to detect the outbreak of an episode. Also, the alarms raised by the system allow clinicians, relatives, or caregivers in general to take a proactive action to start the treatment before the user asks for it.

The current paper presents the MoodRecord system in the framework of the NYMPHA-MD Project [12]. The MoodRecord system identifies signs of deviations in mood based on the fusion of data collected using a smartphone, allowing early interventions. The different data sources captured by the MoodRecord application have been chosen to cover most of the items in the RSs. In this light, the paper describes the methodology used to extract mood with a special focus on video and voice modules as they are the most complex ones.

The paper is structured as follows. Section 2 briefly overviews related applications on automatic mood detection and related works on detection of mood from video and voice. Section 3 describes the MoodRecord mobile application created for patient continuous supervision in the framework of NYMPHA-MD, detailing the implementation of the video and voice modules. Section 4 contains the description of the pilot implementation and evaluation; and finally, Section 5 sketches the final conclusions and foreseen future work.

\section{Related Work}

Automatic home monitoring for any disorder, illness or impairment will be effective if combined with suitable self-monitoring platforms. Recently, monitoring platforms for computers and smartphones have been released (see [26,13] for an overview on the use of smartphones for the detection of medical disorders). The main drawback most of these systems present is the inability of collecting objective data from the behaviour of the patients. Moreover, they usually do not provide any feedback from healthcare providers. Some recent projects such as $\mathrm{PSYCHE}^{5}$ or MONARCA ${ }^{6}[13]$ are specifically related to the detection and treatment of BD. Although they in general put a step forward in the automatic monitoring of bipolar disorder, their main drawbacks are the high level of intrusiveness for patients due to a large number of sensors required, and the inability to target the caregiver's role.

Mood detection from voice. Speech is a powerful identifier for the detection of bipolar disorder, as it has been shown in several works [21,19, 11]. Moreover, voice is a non-intrusive and ubiquitous sensor. The automatic detection of mental disorders - or other disorders that can be reflected in language - can be performed either by means of context-dependent features or acoustic-dependent features $[20,29,3]$. While the former ones require the use of language transcriptions, the latter make use of solely acoustic features extracted from speech. Specifically, the acoustic-based systems rely on the extraction of speech-based features regardless of the language, which makes it a language-independent sys-

\footnotetext{
${ }^{5}$ www.psyche-project.org

${ }^{6}$ www.monarca-project.eu
} 
tem. The acoustic features can be, among others, spectral features, voice quality characteristics, or prosodic features extracted in their acoustic form.

Other studies report the usefulness of prosodic features to detect emotional states such as depression and mania. In [24], for example, several features such as pitch rate, jitter, shimmer and harmonic to noise ratio indices were shown to be indicators for patients with voice disability in contrast to healthy people - especially pitch rate-. In [27], jitter, shimmer and first and second formant frequencies differed significantly in depressed speech. In a similar way, [23] analyses the chaotic behaviour of vocal sounds in patients with depression, and [32] proposes a new feature value based on the nonlinear Teager energy operator to classify speech under stressed conditions. Other voice quality characteristic are further studied in [22] to detect depression and post-traumatic stress disorder, and speech pressure has also been reported as indicator for mania states [2].

Mood detection from video. The automatic analysis of perceived mood estimation through the analysis of human facial expressions (FE) refers to a set of methodologies and studies that relate artificial intelligence with behavioural sciences and neurology. Faces carry a wealth of social information, including information about identity, emotional and motivational cues, lip speech, and focus of attention as indicated by eye gaze, all of which are important for successful communication. Human emotion studies have been mainly described by either a categorical or a dimensional approach. The categorical approach, implemented in this study, maps any perceived emotion into a closed group of predefined FE. Among the existing categorical approaches for FE, Ekman's set of 7 universal expressions + neutral state $[8,9]$ is one of the most used ones (Figure 1). This set includes expressions that provide additional information to the non-invasive evaluation of depression and mania scales in BD. For a wider comprehension on Automatic Facial Expression Recognition (AFER) systems, and its current state-of-the-art methodology for accurate recognition based on deep learning approaches, the reader is referred to [5].

\section{The MoodRecord application}

The NYMPHA-MD Project: Next Generation Mobile Platforms for HeAlth, in Mental Disorders ${ }^{7}$ is an FP7 European project that launched a Pre-Commercial

${ }^{7}$ https://cordis.europa.eu/project/id/610462

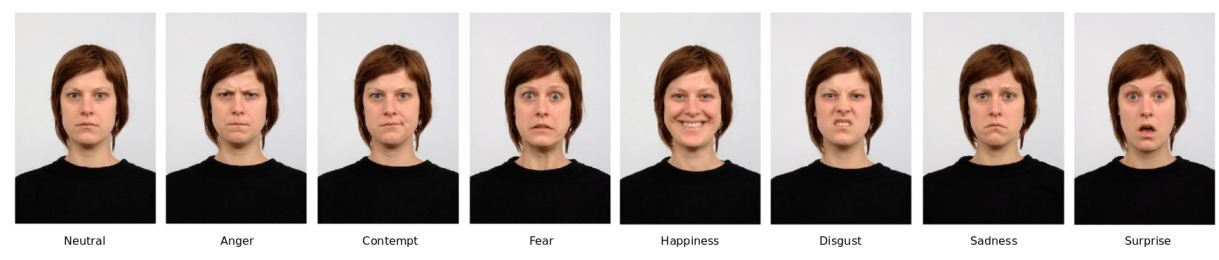

Fig. 1. 7 Ekman core facial expressions + neutral state. Image from [18]. 


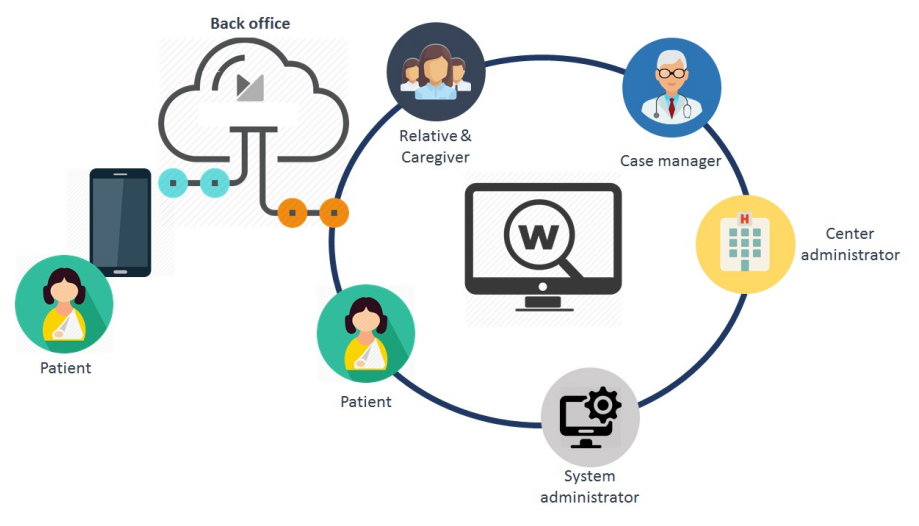

Fig. 2. Diagram of the MoodRecord system architecture showing its main parts (Front End/App, Back End and Back Office/Web) and potential users.

Procurement (PCP) bid to look for solutions on continuous monitoring of bipolar disorder. PULSO Ediciones S.L. led a team with the Universitat Pompeu Fabra and the Center for Computer Vision, in charge of the speech and face analysis modules, respectively. The team participated in the bid with the MoodRecord system, that passed satisfactorily all the eliminating rounds of the PCP (definition, prototype, and pilot).

The MoodRecord application developed according to the technical specifications for NYMPHA-MD project aims to provide a new way to manage patients diagnosed with bipolar disorder, developing a system that keeps an important role to informal caregivers. The system performs patient monitoring to detect and predict changes in patient's mood, maximising the treatment adherence by means of personalised data and feedback under the supervision of clinicians. The MoodRecord system consists of a web interface and a mobile application, both managed from a back-office server. The application is intended to be used by patients suffering BD (primary users) under clinician prescription. The website has been developed to provide clinicians with all data from patients, collected by the app, to simplify and improve their follow up. Patients, relatives and caregivers can access the web using a reduced set of functionalities. Figure 2 shows a diagram of the Mood Record schematic architecture, with the different stakeholders.

The MoodRecord App runs on Android and IOS systems. Figure 3a shows the sidebar menu of the MoodRecord application, with the different actions the user can perform. The application manages a weekly guideline defined by the doctor, with a set actions and questionnaires that the patient must perform. The different actions available for the patient are the following:

- Mood: multiple choice mood selector (Figure 3b).

- Activities: to report the activities performed during the day (Figure 3c). 

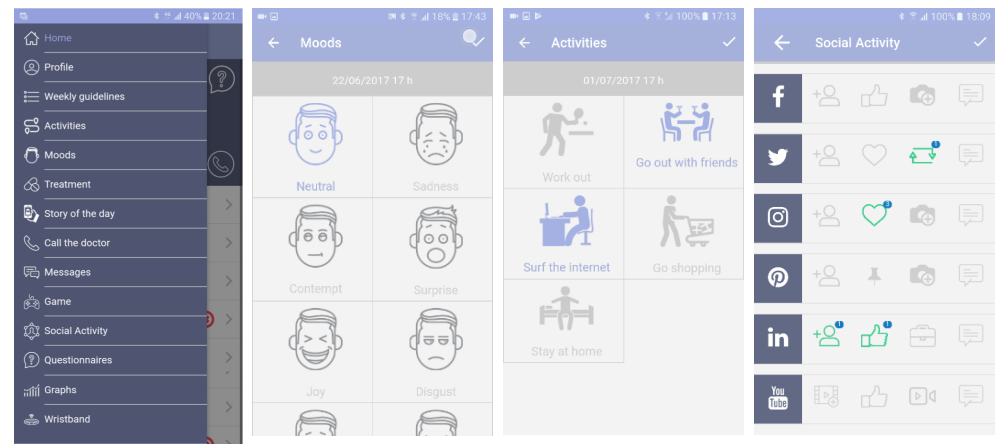

Fig. 3. Screenshots of the MoodRecord application. From left to right: a) main menu, b) mood selection screen, c) activity recording screen and d) social activity screen.

- Story of the day: to self-record their daily experiences, using the frontal camera for later video and audio analysis.

- Treatment: to report compliance to any treatment prescription.

- Social activity: to indicate daily activity on social networks (Figure 3d).

- Questionnaires: to fill out different self-administered RSs available, either planned by doctors or when the system predicts some risk of suicide: Barratt's Impulsivity Scale, Beck Hopelessness Scale, Buss-Durkee Hostility Inventory, Generic health status scale: EQ-5D-5L, Perceived Stress Scale, Patient Health Questionnaire 15 and Personal and Social Performance.

The MoodRecord App includes a game that the user can play at any moment. The system monitors data about the time spent in the game and telemetry (velocity, intensity, attempts, etc.). The application also monitors the application's usage to measure how the patient adheres to the plan. Finally, the application is bound to an activity wristband (FitBit $\AA$ ) to record sleep times and physical activity. The data collected is highly correlated to the different items measured by HDRS and YMRS scales to evaluate bipolarity, and tries to cover the whole evaluation range. Table 1 shows this relationship between the data collected by the App and items in both scales.

The MoodRecord application integrates and reports the data extracted from the different modules and combines the different sources of information to estimate risk of mania or depression. Clinicians use the scales to evaluate patients during visits. Such evaluations are used by MoodRecord to recognize the normal states of the user. In the case where $30 \%$ of the previous week's registers, obtained or computed from any of the different sources, are outside these normality records, the system raises an alarm. The system will also raise an alarm if the scores of the self-administered scales overcome the threshold of "normal values". If the system detects a severe depression risk (indicated by a higher number of registers with a high deviation from the normality values) the system will automatically ask the user to answer the BHS questionnaire, to detect suicide tendencies and raise a severe alarm. 
Table 1. correspondence between items in HDRS and YMRS scales and values measured by MoodRecord

\begin{tabular}{|l|l|l|l|}
\hline Feature & HDRS elements & YMRS elements & MoodRecord \\
\hline Mood & mood & mood & mood, video, voice \\
\hline Activity & work/activities, agitation & motor activity & activity record, wristband \\
\hline Sleep & insomnia & sleep & wristband \\
\hline Speech & retardation, anxiety & speech rate, language & voice \\
\hline Behaviour & agitation & $\begin{array}{l}\text { irritability, disruption, } \\
\text { aggressive }\end{array}$ & voice, game \\
\hline Sex & genital symptoms & sexual interest & social activity \\
\hline Insight & $\begin{array}{l}\text { insight, suicide, guilt feel- } \\
\text { ings }\end{array}$ & insight, paranoid ideas & self-administered scales \\
\hline Other & $\begin{array}{l}\text { somatic symptoms, loss of } \\
\text { weight, hypochondria }\end{array}$ & appearance & \\
\hline
\end{tabular}

\subsection{Mood detection from voice}

For the NYMPHA-MD project hereby presented, we developed a Praat-based [1] module for prosodic feature extraction in order to detect mania, depression and normal states. The features extracted in our module are: mean fundamental frequency (F0), maximum value of F0, minimum F0, range of F0, first formant frequency (F1), second formant frequency (F2), relative jitter, absolute jitter, relative shimmer, absolute shimmer [10], noise-to-harmonic ratio (NHR), harmonic-to-noise ratio (HNR), intensity, number of pauses, speech rate, articulation rate, and average syllable duration. The last four features were computed without using any textual transcriptions by means of the algorithm described in [6].

A set of regression models is trained to identify YMRS and HDRS values. Regression is based on the combination of different models: (a) models that estimate the global HDRS and YMRS values, and (b) models that estimate some HDRS/YMRS items related to speech and language.

For each model, two different versions are combined into a hybrid model: (1) a general model to be used to classify any new patient once her normal status is defined, and (2) a personalized version for each patient. The hybrid system uses the general models until the personalized models outperform the general one. To obtain the personal model, the system uses the data filled by doctors during interviews. After each interview, models are retrained and check for performance.

General models have been trained with data provided by the NYMPHA-MD consortium during the second phase of the project and data collected during the pilot. This dataset is composed of 30 valid recordings with the corresponding evaluations by doctors. Although the dataset is small and most of the recordings were done with users in an euthymic state, the results obtained using support vector regression with a radial kernel showed that, while HDRS estimation achieved the best result by using low-level voice quality and formant features, the best result when estimating YMRS was achieved by using the whole combination of voice quality, formant, and prosodic features [11]. This can be explained 


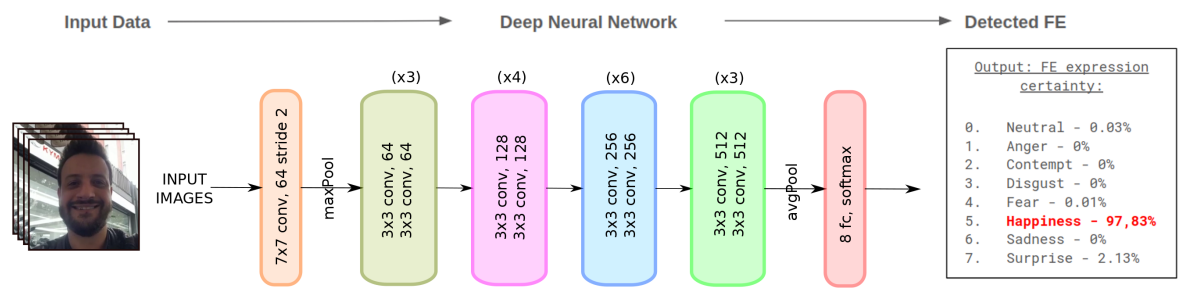

Fig. 4. Adapted ResNet50 neural network as a FE classifier.

by the fact that mania state is more expressive in terms of speech, and thus the need of prosodic information is much more relevant.

\subsection{Mood detection from video}

The NYMPHA-MD project includes an AFER system following Ekman's categorical proposal on emotional analysis over a universal reduced set of FEs, by classifying any FE within an 8-class categorical classifier. Such a system could benefit from existing validated public datasets. The solution adopted is applied over each individual frame independently, and uses a deep learning scheme that tackles: (a) face detection, by means of a Viola-Jones algorithm [28], which works with intensity fluctuation in order to capture faces trained in a near-frontal position from images, shown to be very fast and with a good performance for MoodRecord scenario, and (b) classification problem, by means of a standard deep neural network (Figure 4) that will get the resulting face detected as a RGB input image to convey to a probability vector containing the confidence for each of the 8 possible FEs mentioned before. The predicted expression will be the one with major relevance. The predictions for each frame of the video add a temporal dimension to the analysis. Histograms of frequency can be drawn to compute regular or deviation patterns of apparent mood.

Convolutional neural networks are very robust to image variability such as ethnicity, gender and age, hence performing well under unseen data. The model used (standard ResNet50 architecture, Figure 4) has been fine tuned over an existing pretrained model (ImageNet [7]). After selecting the valid images for

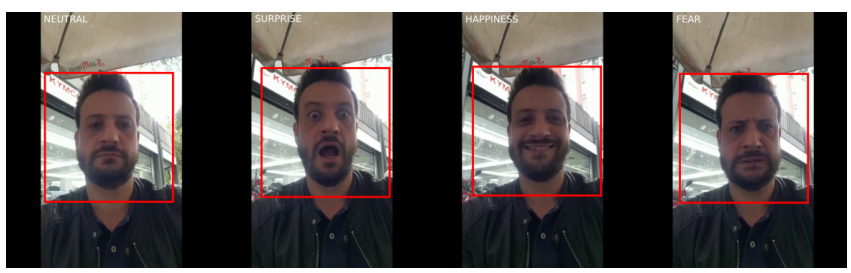

Fig. 5. Visual results from the perceived emotion analysis from video. 


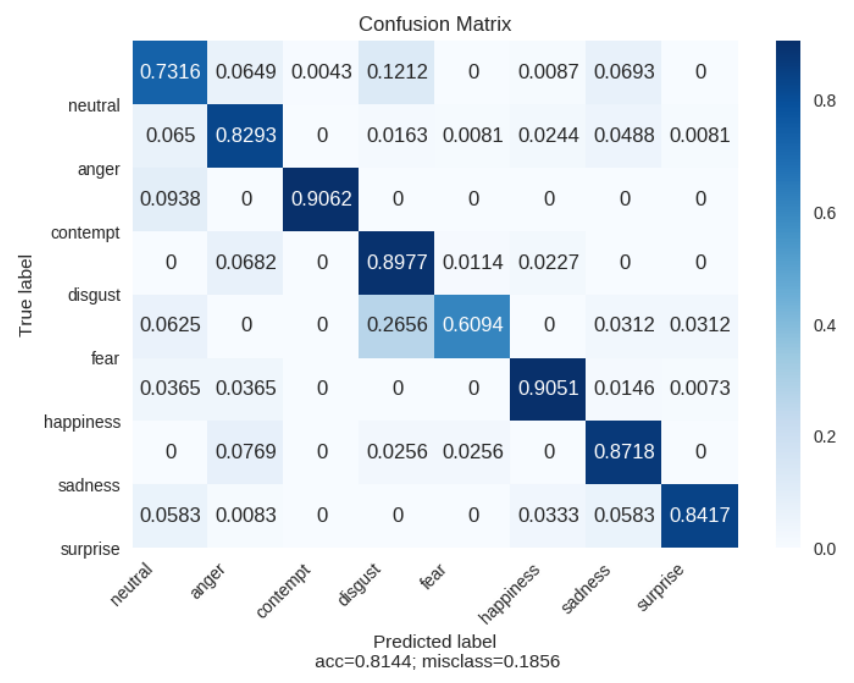

Fig. 6. Confusion matrix from test data.

training, the final set was composed of 8832 images split into the eight categories. Since the dataset was unbalanced towards the neutral expression, additional data was included by manually scraping images from the internet. The dataset was divided in 70/20/10\% (train/validation/test) splits. Once trained, the complete face analysis pipeline (face detector + facial expression classifier) was tested over real unseen data. Figure 5 shows a visual performance over a few testing examples: the system is capable of detecting near-frontal face area of interest (red bounding box), which is later passed to the facial expression perceived mood module, that outputs the perceived mood (top left corner from each of the samples shown in Figure 5). Under semi-controlled environments (limited range of head pose angles, illumination and image quality) the system is capable of detecting the different FEs from a subject with over $90 \%$ accuracy in most of the classes (see confusion matrix in Figure 6).

\section{Pilot testing}

During the last phase of the NYMPHA-MD project, MoodRecord system was used on a pilot to measure the usability and feasibility of the application. The full pilot was run from January 2018 to March 2018, but the patients follow-up took 4 weeks, and it was conducted by the three institutions in charge of the project: the Mental Health Department of the Azienda Provinciale per i Servizi Sanitari (Trento, Italy), Corporació Sanitària Parc Taulí (Sabadell, Catalonia), and The Psychiatric Center Copenhagen (Copenhagen, Denmark).

The study includes 30 patients (10 on each location) aged 18 to 65 years old with a bipolar disorder diagnosis according to ICD-10 using Schedules for Clinical 
Assessments in Neuropsychiatry [30]. Following inclusion, baseline assessments were conducted on all patients to check that they were not in an episode. To adjust and personalise the system, each participant had to be in a normal state to start the monitoring, thus the system has a personalised "reference status" to compare with. The study was designed and powered to investigate differences in symptoms and functioning due to using a smartphone-based monitoring system. Once a day the patients should enter and evaluate mood, activity level, enter a story of the day, whether medicine was intake and activity level on social media. The main focus of the study was to measure the system's usability and feasibility performance. The users rated the system, according to the USE and CSUQ scales with positive evaluations (average 4.4 out of 7 ). Even there where significant differences between users from Denmark and the ones from Italy or Spain that showed a similar criteria. Interestingly, audio and visual estimation provided consistent, correlated, and regular recognition patterns of mood for the patients, as verified by the physicians and possibly because of the no existence of episodes during the pilot.

Nowadays there is an undergoing process to fulfil the administrative steps required to get the CE Medical Certification (class type I or IIa) of the system. A prerequisite to be installed on different hospitals, that have already shown their interest in the product.

\section{Conclusions and future work}

We have presented MoodRecord, a system to monitor patients with Bipolar Disorder for early detection of relapses. The system includes an app for smartphones that collects data of different modes, which include the analysis patient's voice and face, to report the mood status so that clinicians can take the appropriate actions before the patient worsens.

The system is fully operative and has been tested in a pilot use case where patients have approved its usability. The vision system has shown a good performance in classification of facial expressions with external data and correlation with patient reports during the pilot. The voice analysis has shown consistent results with testing data using quality, formant and prosodic features.

The future of the application depends on obtaining the CE Medical Certificate that will allow to install the application in hospitals (there are already some interested in the product). This usage will produce more data from users and evaluations from clinicians, that will allow to fine tune the voice and image models to increase their performance and reduce possible bias under different kinds of population underrepresented during testing.

\section{Acknowledgements}

This work is part of the MYMPHA-MD project, which has been funded by the European Union under Grant Agreement No 610462. It has also been partially supported by the Spanish project PID2019-105093GB-I00 (MINECO/FEDER, 
UE) and CERCA Programme/Generalitat de Catalunya.), and by ICREA under the ICREA Academia programme. The last author has been funded by the Agencia Estatal de Investigación (AEI), Ministerio de Ciencia, Innovación y Universidades and the Fondo Social Europeo (FSE) under grant RYC-201517239 (AEI/FSE, UE). The authors would like to thank Ivan Latorre for his technical support and Giorgia Cistola for her help on the data preparation.

\section{References}

1. Boersma, P.: Praat: doing phonetics by computer [computer program]. http://www. praat. org/ (2020)

2. Carlson, G.A., Goodwin, F.K.: The stages of mania: A longitudinal analysis of the manic episode. Archives of General Psychiatry 28(2), 221-228 (1973)

3. Chien, Y.W., Hong, S.Y., Cheah, W.T., Yao, L.H., Chang, Y.L., Fu, L.C.: An automatic assessment system for Alzheimer's disease based on speech using feature sequence generator and recurrent neural network. Scientific Reports 9(1), 1-10 (2019)

4. Colom, F., Lam, D.: Psychoeducation: Improving outcomes in bipolar disorder. European Psychiatry 20(5), 359-364 (2005)

5. Corneanu, C., Oliu, M., Cohn, J., Escalera, S.: Survey on RGB, 3D, thermal, and multimodal approaches for facial expression recognition: History, trends, and affect-related applications. IEEE Transactions on Pattern Analysis and Machine Intelligence 38, 1-1 (2016)

6. De Jong, N.H., Wempe, T.: Praat script to detect syllable nuclei and measure speech rate automatically. Behavior research methods 41(2), 385-390 (2009)

7. Deng, J., Dong, W., Socher, R., Li, L.J., Li, K., Fei-Fei, L.: ImageNet: A large-scale hierarchical image database. In: CVPR09 (2009)

8. Ekman, P.: Universals and Cultural Differences in Facial Expressions of Emotions. Lincoln, NB: University of Nebraska Press (1971)

9. Ekman, P., Oster, H.: Facial expressions of emotion. Annual Review of Psychology 30(1), 527-554 (1979)

10. Farrús, M., Hernando, J., Ejarque, P.: Jitter and shimmer measurements for speaker recognition. In: Proceedings of the Interspeech (2007)

11. Farrús, M., Codina-Filbà, J., Escudero, J.: Acoustic and prosodic information for home monitoring of bipolar disorder. Health Informatics Journal (2021)

12. Faurholt-Jepsen, M.: The NYMPHA-MD project: Next generation mobile platforms for health, in mental disorders. European Psychiatry 41(S1), S23-S23 (2017)

13. Gravenhorst, F., Muaremi, A., Bardram, J., Grünerbl, A., Mayora, O., Wurzer, G., Frost, M., Osmani, V., Arnrich, B., Lukowicz, P., et al.: Mobile phones as medical devices in mental disorder treatment: an overview. Personal and Ubiquitous Computing 19(2), 335-353 (2015)

14. Hamilton, M.: The hamilton rating scale for depression. In: Assessment of depression, pp. 143-152. Springer (1986)

15. Joyce, P.R.: Illness behaviour and rehospitalization in bipolar affective disorder. Psychological Medicine 15(3), 521-525 (1985)

16. Lam, D., Wong, G.: Prodromes, coping strategies, insight and social functioning in bipolar affective disorders. Psychological Medicine 27(5), 1091-1100 (1997)

17. Lam, D., Wong, G.: Prodromes, coping strategies and psychological interventions in bipolar disorders. Clinical Psychology Review 25(8), 1028-1042 (2005) 
18. Langner, O., Dotsch, R., Bijlstra, G., Wigboldus, D., Hawk, S., Knippenberg, A.: Presentation and validation of the radboud face database. Cognition \& Emotion 24, 1377-1388 (12 2010)

19. Maxhuni, A., Muñoz-Meléndez, A., Osmani, V., Perez, H., Mayora, O., Morales, E.F.: Classification of bipolar disorder episodes based on analysis of voice and motor activity of patients. Pervasive and Mobile Computing 31, 50-66 (2016)

20. Mota, N.B., Vasconcelos, N.A., Lemos, N., Pieretti, A.C., Kinouchi, O., Cecchi, G.A., Copelli, M., Ribeiro, S.: Speech graphs provide a quantitative measure of thought disorder in psychosis. PloS one 7(4), e34928 (2012)

21. Muaremi, A., Gravenhorst, F., Grünerbl, A., Arnrich, B., Tröster, G.: Assessing bipolar episodes using speech cues derived from phone calls. In: International Symposium on Pervasive Computing Paradigms for Mental Health. pp. 103-114. Springer (2014)

22. Scherer, S., Stratou, G., Gratch, J., Morency, L.P.: Investigating voice quality as a speaker-independent indicator of depression and PTSD. In: Interspeech. pp. 847$851(2013)$

23. Shimizu, T., Furuse, N., Yamazaki, T., Ueta, Y., Sato, T., Nagata, S.: Chaos of vowel/a/in japanese patients with depression: a preliminary study. Journal of Occupational Health 47(3), 267-269 (2005)

24. Shinohara, S., Nakamura, M., Mitsuyoshi, S., Tokuno, S., Omiya, Y., Hagiwara, N.: Voice disability index using pitch rate. In: IEEE EMBS Conference on Biomedical Engineering and Sciences (IECBES). pp. 557-560 (2016)

25. Strakowski, S.M., DelBello, M.P., Adler, C.M., Fleck, D.E.: Bipolar Disorder. Oxford University Press, USA (2020)

26. Torous, J., Powell, A.C.: Current research and trends in the use of smartphone applications for mood disorders. Internet Interventions 2(2), 169-173 (2015)

27. Vicsi, K., Sztahó, D., Kiss, G.: Examination of the sensitivity of acoustic-phonetic parameters of speech to depression. In: IEEE 3rd International Conference on Cognitive Infocommunications (CogInfoCom). pp. 511-515 (2012)

28. Viola, P., Jones, M.: Robust real-time object detection. In: International Journal of Computer Vision (2001)

29. Voleti, R., Woolridge, S., Liss, J.M., Milanovic, M., Bowie, C.R., Berisha, V.: Objective assessment of social skills using automated language analysis for identification of schizophrenia and bipolar disorder. arXiv preprint arXiv:1904.10622 (2019)

30. Wing, J.K., Babor, T., Brugha, T., Burke, J., Cooper, J., Giel, R., Jablenski, A., Regier, D., Sartorius, N.: SCAN: Schedules for Clinical Assessment in Neuropsychiatry. Archives of General Psychiatry 47(6), 589-593 (1990)

31. Young, R.C., Biggs, J.T., Ziegler, V.E., Meyer, D.A.: A rating scale for mania: reliability, validity and sensitivity. The British journal of psychiatry 133(5), 429435 (1978)

32. Zhou, G., Hansen, J.H., Kaiser, J.F.: Nonlinear feature based classification of speech under stress. IEEE Transactions on speech and audio processing $\mathbf{9}(3)$, 201216 (2001) 